\title{
Video Article \\ Flash NanoPrecipitation for the Encapsulation of Hydrophobic and Hydrophilic Compounds in Polymeric Nanoparticles
}

\author{
Chester E. Markwalter ${ }^{1}$, Robert F. Pagels ${ }^{1}$, Brian K. Wilson ${ }^{1}$, Kurt D. Ristroph ${ }^{1}$, Robert K. Prud'homme ${ }^{1}$ \\ ${ }^{1}$ Department of Chemical and Biological Engineering, Princeton University
}

Correspondence to: Robert K. Prud'homme at prudhomm@princeton.edu

URL: https://www.jove.com/video/58757

DOI: doi:10.3791/58757

Keywords: Bioengineering, Issue 143, Nanoparticle, micromixing, block copolymer, drug delivery, peptide, protein, process scale-up

Date Published: 1/7/2019

Citation: Markwalter, C.E., Pagels, R.F., Wilson, B.K., Ristroph, K.D., Prud'homme, R.K. Flash NanoPrecipitation for the Encapsulation of Hydrophobic and Hydrophilic Compounds in Polymeric Nanoparticles. J. Vis. Exp. (143), e58757, doi:10.3791/58757 (2019).

\section{Abstract}

The formulation of a therapeutic compound into nanoparticles (NPs) can impart unique properties. For poorly water-soluble drugs, NP formulations can improve bioavailability and modify drug distribution within the body. For hydrophilic drugs like peptides or proteins, encapsulation within NPs can also provide protection from natural clearance mechanisms. There are few techniques for the production of polymeric NPs that are scalable. Flash NanoPrecipitation (FNP) is a process that uses engineered mixing geometries to produce NPs with narrow size distributions and tunable sizes between 30 and $400 \mathrm{~nm}$. This protocol provides instructions on the laboratory-scale production of core-shell polymeric nanoparticles of a target size using FNP. The protocol can be implemented to encapsulate either hydrophilic or hydrophobic compounds with only minor modifications. The technique can be readily employed in the laboratory at milligram scale to screen formulations. Lead hits can directly be scaled up to gram- and kilogram-scale. As a continuous process, scale-up involves longer mixing process run time rather than translation to new process vessels. NPs produced by FNP are highly loaded with therapeutic, feature a dense stabilizing polymer brush, and have a size reproducibility of $\pm 6 \%$.

\section{Video Link}

The video component of this article can be found at https://www.jove.com/video/58757/

\section{Introduction}

Since the late 1990s, there has been a steady increase in the number of clinical trials employing nanomaterials ${ }^{1,2}$. The rising interest reflects the promise of nanomaterials to improve the bioavailability of hydrophobic drugs and to enable preferential targeting within the body ${ }^{3}$. Polymeric nanoparticles (referred to as nanoparticles or NPs here) represent a growing proportion of this class of materials ${ }^{2}$. NPs have garnered interest because they have highly tunable properties such as size, composition, and surface functionalization ${ }^{4}$. When applied to the administration of poorly soluble drugs, NPs frequently have a core-shell structure where the therapeutic is encapsulated in the hydrophobic core and the shell consists of a hydrophilic polymer brush. A simple way to generate this structure employs an amphiphilic diblock copolymer (BCP) consisting of a degradable hydrophobic block, which forms part of the particle core, and a hydrophilic poly(ethylene glycol) (PEG) block, which forms the polymer brush and imparts steric stabilization ${ }^{4,5}$.

Nanoprecipitation is a common fabrication technique for polymeric nanoparticles because it is simple and not energy intensive ${ }^{6}$. In its simplest form, nanoprecipitation involves addition by pipette of NP components in an organic solvent like acetone to an excess volume of stirred water. The change in solvent to a dilute aqueous solution results in the precipitation of the insoluble core component. The stabilizer assembles on this growing particle surface, directed by adsorption of the collapsed hydrophobic block ${ }^{7,8,9,10}$. A uniform particle size distribution is obtained when the solvent and water rapidly mix to form a homogeneous solution. Mixing that is slower than the nucleation and assembly of the components results in a larger, more polydisperse particle population. Though readily accessible for a simple test, the stirred batch approach results in wide variability due to mixing inconsistency and is not amenable to scale-up ${ }^{6,11}$. Microfluidics have emerged as another avenue to NP production that can be run continuously. This means of production has been recently reviewed by Ding et al. ${ }^{11}$. A common approach uses laminar flow focusing to reduce the solvent length scale to sub-micron values. Mixing of the antisolvent occurs by diffusion, so small flow dimensions are crucial to ensure uniform particles ${ }^{11,12}$. Parallelization of multiple microfluidic chambers for scale-up is problematic for large production volumes.

The rapid mixing conditions that favor uniform nanoprecipitation in microfluidics alternately can be produced in confined, turbulent flows. Flash NanoPrecipitation (FNP) employs special mixing geometries to achieve these conditions under higher volumetric flowrates than possible with microfluidics. Inlet streams enter a mixing chamber under turbulent conditions that lead to the generation of vortices, so that solvent/anti-solvent lamellae form on the length scale of diffusion ${ }^{11,13}$. Thus, uniform mixing on a time scale shorter than nucleation and growth of the therapeutic is achieved. The confined geometry of the mixer does not permit stream bypassing of the region where turbulent energy dissipation occurs and the entire system experiences the same process history ${ }^{13}$. Nucleation occurs uniformly in the mixing chamber and particle growth proceeds until halted by the assembly of the BCP onto the surface ${ }^{9,14}$. The mixed stream containing stable particles may then be diluted with additional antisolvent to suppress Ostwald ripening of the particles ${ }^{15,16,17}$ 
A confined impinging jet $(\mathrm{CIJ})$ mixer is the simplest mixer design for FNP and permits mixing of two streams in a scalable and continuous fashion, as shown in Figure $1 \mathbf{A}^{13}$. A multi-inlet vortex mixer (MIVM) was developed to enable up to four different stream inputs while still achieving the rapid micromixing required for uniform particle formation, as shown in Figure $1 \mathrm{~B}^{18}$. FNP enables simple formulation screening that can readily be translated to commercial-scale production. Due to the continuous nature of the process, larger batch sizes do not require new vessels but rather longer run times, enabling simple translation to kilogram-scale production in the same equipment train.

Hydrophilic compounds such as peptides and proteins ('biologics') can also be encapsulated in a process termed inverse Flash NanoPrecipitation (iFNP). The technique requires an amphiphilic BCP where one block is hydrophobic and the other is a polyacid ${ }^{19}$. The initial step involves rapid mixing of a dimethyl sulfoxide (DMSO) stream containing the biologic and the BCP against a lipophilic solvent such as dichloromethane or chloroform. This results in the formation of particles stabilized with the hydrophobic block brush. Here, such an architecture will be termed an 'inverted' NP. The core contains the polyacid, which is then ionically crosslinked using a multivalent cation. This stabilizes the particles for processing into an aqueous environment in the form of microparticles or PEG-coated nanoparticles by techniques that have been reported in the literature ${ }^{19,20,21}$.

This protocol can be employed for the lab-scale production of polymeric core-shell nanoparticles encapsulating either hydrophobic or hydrophilic compounds. The subsections of the protocol provide instructions on the use of both mixer classes - the ClJ and the MIVM. The reader should be able to adapt the protocol for novel core components and reproducibly generate nanoparticles of a desired size using the appropriate mixer for the stream inputs. Three example formulations using FNP and iFNP are presented below. Two employ the CIJ mixer and one requires the MIVM $^{15,22}$. The first formulation demonstrates encapsulation of a model hydrophobic compound by FNP. The second formulation demonstrates encapsulation of a model hydrophilic compound by iFNP in a CIJ mixer. The final formulation provides an example of protein encapsulation by iFNP using a MIVM. The protocol for this third formulation describes the use of a small-scale, handheld MIVM termed the ' $\mu$ MIVM.' The mixer design is smaller to allow for simplified formulation screening, but the scaling behavior is well understood and the mixer is not a microfluidic device $^{22}$. The final section of the protocol includes some notes on scale-up of lead formulations identified in screening. These formulations are intended to provide access points for learning the process and consequently use non-degradable poly(styrene)-based polymers. Alternative stabilizers have been described in the literature, with a number of biocompatible commercial options available ${ }^{14,23,24}$.

\section{Encapsulation of Hydrophobic Compounds in Polymeric NPs Using a ClJ Mixer}

1. Prepare and clean equipment.

1. Procure and validate a CIJ mixer.

Note: See Supplemental Information section 1 for construction guidance. CAD files are available as Supplemental Information as well.

2. Before each use, ensure that all fittings on the CIJ mixer are snug and the outlet tubing is not bent or pinched.

3. In a fume hood, attach a $5 \mathrm{~mL}$ luer lock syringe containing $2-3 \mathrm{~mL}$ of solvent to each inlet adapter. Select a solvent (e.g., acetone) that will clean any compounds recently used in the mixer.

Note: Typical selections are acetone or tetrahydrofuran (THF). Only use polypropylene syringes to avoid solvent compatibility problems such as leaching. Do not use syringes with rubber O-ring seal plungers.

4. Set the $\mathrm{ClJ}$ assembly over a waste container.

Note: A flask with an opening smaller than the CIJ body works well as this supports the mixer and allows easy operation of the syringes.

5. Steadily depress the syringe plungers to empty the contents through the mixing chamber over a few seconds. Remove the syringes. Note: Syringes can be retained and reused for multiple rounds of cleaning between FNP runs.

6. Dry the CIJ mixer internals using a $\mathrm{N}_{2}$ stream. A male luer adapter on the end of a $\mathrm{N}_{2}$ line is effective.

Note: If the cleaning solvent is not volatile (e.g., DMSO), repeat steps 1.1.3-1.1.5 with acetone or THF before proceeding to step 1.1.6. It is crucial to remove residual solvent for run-to-run consistency.

2. Prepare solvent and antisolvent streams at target compositions.

1. Dissolve the hydrophobic compound (i.e., vitamin $\mathrm{E}$ ) in unstabilized THF at $10 \mathrm{mg} / \mathrm{mL}$ in sufficient amount to complete the desired number of FNP runs. Prepare slightly more than needed per run.

Note: Other solvents can be used in these steps, subject to the constraints in the Discussion section. If employing THF, stabilizerfree solvent is recommended because butylated hydroxytoluene has low aqueous solubility. Use caution to avoid peroxide buildup (including peroxide testing) and be aware that low levels of peroxides may interfere with certain NP applications (e.g., bleaching of dyes).

2. Mix the vitamin E solution on a vortex mixer until dissolved.

Note: For some compounds, bath sonication for 1-2 min may assist in generating a dissolved solution. It is important that all NP components are molecularly dissolved.

3. Dissolve the block copolymer stabilizer (i.e., poly(styrene)-b-poly(ethylene glycol), $\mathrm{PS}_{1.6 \mathrm{k}}-b-\mathrm{PEG}_{5 \mathrm{k}}$ ) in THF at $10 \mathrm{mg} / \mathrm{mL}$ at approximately the same volume as in step 1.2.1 to form the polymer solution.

Note: Other solvents can be used, subject to the constraints detailed in the Discussion section.

4. Mix the polymer solution with a vortex mixer until dissolved. If necessary, place the solution in a sonication bath for 1-2 min to aid in solids dissolution.

Note: The polymer cannot be in a micellar form. Dynamic light scattering (DLS) can be a useful tool to determine whether a new stream composition meets this criterion.

5. Create the solvent input stream containing $5 \mathrm{mg} / \mathrm{mL}$ of both vitamin $E$ and the stabilizer ( $50 \%$ vitamin $E$ loading) by first pipetting 0.25 $\mathrm{mL}$ of the vitamin $\mathrm{E}$ solution into a $1.5 \mathrm{~mL}$ centrifuge tube. Then pipette $0.25 \mathrm{~mL}$ of the polymer solution into the same tube. Note: Volumes larger than $0.5 \mathrm{~mL}$ per run are feasible with different syringe sizes. Above $10 \mathrm{~mL}$ input volume, it is practical to use a syringe pump. 
6. Mix well on a vortex mixer for 5-10 s. Optionally, centrifuge the tube at $1000 \mathrm{xg}$ for 5-10 s to recover any liquid stuck to the cap, which improves reproducibility between CIJ runs.

7. Pipette $0.525 \mathrm{~mL}$ of deionized water into a second $1.5 \mathrm{~mL}$ centrifuge tube as the antisolvent stream.

Note: It is better to have excess antisolvent, which ensures that the solvent stream never enters the mixing chamber without antisolvent present. In some cases where salt solubility in the solvent/antisolvent mixture is not limiting, buffered aqueous systems can be used.

8. Pipette $4 \mathrm{~mL}$ of deionized water into a $20 \mathrm{~mL}$ scintillation vial or other suitable container as a quench bath. Place a small magnetic stir bar in the vial.

Note: The quench bath reduces the Ostwald ripening by lowering the final solvent content to $10 \%$ by volume ${ }^{15,17}$. This volume may be adjusted to address process constraints and can be directly scaled with input stream volume.

3. Produce NPs by FNP using the CIJ mixer.

1. Position the open quench bath vial below the cleaned CIJ mixer on a stir plate in a fume hood. A practical configuration uses a 50 $\mathrm{mL}$ test tube rack block to support the $\mathrm{CIJ}$ mixer with the vial below and the outlet tubing directed into the vial. See Figure 1A for orientation.

2. Begin stirring the quench bath via the magnetic stir bar at around $75 \%$ max speed.

3. Using a $1 \mathrm{~mL}$ polypropylene syringe fitted with a blunt-tip needle, draw the full volume from the antisolvent tube. Note: Do not use syringes that contain a rubber O-ring seal to avoid compatibility concerns. For larger inlet volumes, use an appropriately sized luer lock syringe. The syringe outlet must be centered on the syringe axis or it will be unstable during depression.

4. Carefully remove all air bubbles from the syringe and remove the blunt tip needle, disposing in a sharps container.

5. Prime the plunger so that the stream comes just to the syringe opening. Attach the syringe to one of the $\mathrm{ClJ}$ inlet fittings.

6. Repeat steps 1.3.3-1.3.5 for the solvent solution.

7. Rapidly, smoothly, and uniformly depress the syringes at the same time by placing the ball of the hand, the palm of the hand, or one thumb each on the tops of the plungers depending on personal preference. Collect the effluent in the quench bath vial. Note: A $0.5 \mathrm{~mL}$ input should be depressed in less than $0.5 \mathrm{~s}$.

8. Set aside the CIJ mixer with the syringes still attached. Remove the stir bar and cap the vial, which now contains the NP dispersion with a core-shell particle structure (Figure 1C).

9. Hold the mixer over a waste solution container and remove the syringes. The hold-up volume (about $0.25 \mathrm{~mL}$ ) will then drain out. Dispose of the used syringes and repeat the cleaning step 1.1 before the next FNP trial.

Note: Do not allow the hold-up volume to empty into the vial containing the NPs as this will negatively impact sample uniformity.

4. Perform analysis and post-processing of NP dispersion.

1. To characterize the NP size using DLS, pipette $100 \mu \mathrm{L}$ of the NP dispersion into a plastic cuvette and add $900 \mu \mathrm{L}$ of the quench bath solvent (e.g., water). Note: Smaller volumes may be used for low-volume cuvettes. A 10 -fold dilution is generally sufficient.

2. Mix well by pipetting up and down or by mild shaking. Follow the instrument-specific instructions to analyze the sample. Note: Alternative characterization techniques such as zeta potential analysis or electron microscopy may be carried out as required. The NP dispersion can be processed further as dictated by the application and reviewed in the Discussion section.

\section{Encapsulation of Hydrophilic Compounds in Inverted NPs Using a CIJ Mixer}

1. Prepare solvent, antisolvent, and quench solutions in fume hood.

1. Complete the cleaning and preparation procedures described in step 1.1, using DMSO as a cleaning solvent and adhering to the note in step 1.1.6 to complete a second rinse with THF.

2. Dissolve the hydrophilic compound (i.e., maltodextrin (MD) with dextrose equivalent (DE) of 4-7, average molecular weight $=3,275 \mathrm{~g} /$ $\mathrm{mol}$, "3k MD") in DMSO at $10 \mathrm{mg} / \mathrm{mL}$ in sufficient volume to complete the desired number of FNP runs.

Note: Other solvents can be used, subject to the constraints outlined in the Discussion section.

3. Mix the maltodextrin solution with a vortex mixer until dissolved. If necessary, place the solution in a sonication bath for 1-2 min to aid in solids dissolution.

4. Create a block copolymer stabilizer (i.e., poly(styrene)-b-poly(acrylic acid), $\mathrm{PS}_{5 \mathrm{k}}-b-\mathrm{PAA}_{4.8 \mathrm{k}}$ ) stock solution in THF at $11.1 \mathrm{mg} / \mathrm{mL}$ at approximately the same volume as in step 2.1.2 to form the polymer solution.

Note: Other solvents and stabilizer concentrations can be used. DMSO can readily be used as a solvent in place of THF.

5. Mix the polymer solution with a vortex mixer until dissolved. If necessary, place the solution in a sonication bath for 1-2 min to aid in solids dissolution.

Note: The polymer input cannot be in a micellar form. DLS can be used to determine whether a new stream composition meets this criterion.

6. Prepare the solvent stream input $(0.5 \mathrm{~mL})$ by combining the following, in order, in a $1.5 \mathrm{~mL}$ centrifuge tube: $0.250 \mathrm{~mL}$ of the $3 \mathrm{k} \mathrm{MD}$ solution, $0.225 \mathrm{~mL}$ of polymer solution, and $0.025 \mathrm{~mL}$ deionized water.

Note: The water content of this stream has a strong impact on NP size and polydispersity. Generally it is best to operate in the $2.5-10$ vol\% range ${ }^{20}$. Values on the high end of the range may help encapsulation of larger molecular weight compounds.

7. Mix well on a vortex mixer for 5-10 s.

8. Optionally, centrifuge the tube at $1000 \times \mathrm{g}$ for $5-10 \mathrm{~s}$ to recover any liquid stuck to the cap, which improves reproducibility between $\mathrm{ClJ}$ runs.

9. Prepare a crosslinker solution of calcium chloride $\left(\mathrm{CaCl}_{2}\right)$ dihydrate in methanol at $25.0 \mathrm{mg} / \mathrm{mL}$.

Note: The crosslinker will be added at a 1:1 charge ratio to the acid groups in the PAA block. Adjust the concentration accordingly if a different crosslinker is used or if a different PAA block size or polymer concentration is used ${ }^{20,21}$.

10. Prepare the antisolvent stream by pipetting $0.5 \mathrm{~mL}$ of chloroform and $0.05 \mathrm{~mL}$ of the crosslinker solution $(0.55 \mathrm{~mL}$ total) into a microcentrifuge tube. 
Note: Other acceptable antisolvents are dictated by the block copolymer choice and typically include dichloromethane or acetone. The crosslinker may instead be added to the quench bath, with additional aging of the NP dispersion to allow for crosslink formation ${ }^{20}$.

11. Mix well on a vortex mixer for 5-10 s.

12. Optionally, centrifuge the tube at $1000 \mathrm{xg}$ for 5-10 s to recover any liquid stuck to the cap, which improves reproducibility between $\mathrm{ClJ}$ runs.

13. Add $4 \mathrm{~mL}$ of the antisolvent (i.e., chloroform) to a $20 \mathrm{~mL}$ scintillation vial to form the quench bath. Place a small magnetic stir bar in the vial.

Note: This volume may be adjusted to address process constraints.

2. Complete the protocol for NP formation as described in step 1.3.

3. Perform analysis and post-processing of NP dispersion.

1. To characterize the NP size using DLS, pipette $100 \mu \mathrm{L}$ of the NP dispersion into a glass cuvette and add $900 \mu \mathrm{L}$ of the solvent used for the quench bath.

2. Mix well by pipetting up and down or by light agitation of the cuvette. Follow the software instructions to analyze the sample. Note: Crosslinking of the NPs can be qualitatively assessed by DLS using a good solvent like DMSO or dimethylformamide (DMF) as DLS diluent ${ }^{20}$. Particles which are stably crosslinked will exhibit an autocorrelation function in the solvent with minimal change in particle size. Poorly crosslinked particles swell and exhibit a weak autocorrelation function and scattering strength ${ }^{21}$.

3. Optionally, add a base, such as ammonia, to drive ionic complexation and strengthen crosslinking in the particle core.

1. Optionally, prepare a $3.48 \mathrm{mg} / \mathrm{mL}$ solution of ammonia in methanol gravimetrically using ammonium hydroxide solution (typically, $30 \mathrm{wt} \%$ ammonia). Add $50 \mu \mathrm{L}$ (i.e., 0.6 equivalents with respect to the acid groups on the polymer) dropwise with stirring Note: The equivalents can be adjusted if desired by varying either the concentration or the volume added ${ }^{25}$.

2. Optionally, age no less than 30 min with mild stirring for crosslinking to occur.

4. Process the NP dispersion to produce either microparticles or coated NPs as described in the literature ${ }^{19,20,21 .}$

\section{Encapsulation of Ovalbumin in Inverted NPs Using a $\mu$ MIVM}

1. Prepare solvent and antisolvent solutions.

1. Prepare a $50 \mathrm{mg} / \mathrm{mL}$ solution of ovalbumin in deionized water ("OVA").

2. Prepare $0.75 \mathrm{~mL}$ of Solution $\mathrm{A}$ in a $1.5 \mathrm{~mL}$ centrifuge tube by diluting $75 \mu \mathrm{L}$ of the OVA solution with $0.675 \mathrm{~mL}$ of DMSO to generate a 5 $\mathrm{mg} / \mathrm{mL}$ solution of OVA in DMSO containing $10 \%$ water by volume. Mix well and centrifuge briefly as described previously. Note: See step 2.1.6 regarding water effects. As in previous sections, the solution volumes can be scaled up or down to fit material needs.

3. Prepare solution $B$ by dissolving the block copolymer stabilizer (i.e., poly(styrene)-b-poly(acrylic acid), $\mathrm{PS}_{5 \mathrm{k}}-b-\mathrm{PAA} \mathrm{A}_{4.8 \mathrm{k}}$ ) in $\mathrm{DMSO}$ at 6 $\mathrm{mg} / \mathrm{mL}$. Mix well and sonicate to dissolve if needed. Pipette $0.75 \mathrm{~mL}$ into a $1.5 \mathrm{~mL}$ centrifuge tube.

4. Pipette $0.75 \mathrm{~mL}$ of THF (Solution $\mathrm{C}$ ) into a $1.5 \mathrm{~mL}$ centrifuge tube.

5. Pipette $1.85 \mathrm{~mL}$ of chloroform (Solution $\mathrm{D}$ ) into a glass scintillation vial.

6. Prepare a $60.0 \mathrm{mg} / \mathrm{mL}$ calcium chloride dihydrate crosslinker solution in methanol. Mix using a vortex mixer.

7. Prepare a $4.17 \mathrm{mg} / \mathrm{mL}$ ammonia solution in methanol as described in step 2.3.4.

8. Add $5.25 \mathrm{~mL}$ of chloroform to a $15 \mathrm{~mL}$ centrifuge tube as the quench bath.

2. Prepare mixer assembly and stand.

1. Gather the bottom receiver, mixing geometry disk, the top disk, the spanner wrench, and an O-ring. See Figure 2 for schematic of components and mixer stand terminology.

Note: Details on MIVM construction may be found in Supplemental Information (Section 1) and in the literature ${ }^{22}$. CAD files are available as Supplemental Information as well.

2. Place the O-ring into the groove, ensuring that it fits well and that there are no signs of wear or damage. Note: Normal operation will lead to worn or solvent-swollen O-rings. If the O-ring appears stretched or deformed, allow it to air dry overnight before use. If the shape does not recover overnight, dispose of the O-ring. Keep a large stock, as this is a consumable part.

3. Carefully align the mixing disk holes with the pegs on the top disk and push together. Ensure that the O-ring does not become displaced by checking the two pieces sit flush.

4. Invert the two pieces and manually assemble them with the bottom receiver. Ensure that the outlet tubing fitting has been loosened so that it does not interfere with complete tightening of the disk.

Note: If the threading catches during assembly, carefully disassemble and apply a food- or pharmaceutical-grade anti-seize to the threading to prevent galling.

5. After manual tightening, fit the spanner wrench to the top disk pegs and snugly tighten the assembly. Then tighten the outlet tubing fitting so that it sits firmly against the bottom face of the mixing geometry. Ensure that the syringe fittings on the top disk are snug.

6. Place the assembled mixer onto the mixer stand so that the outlet tubing extends below the support plate. Support the mobile plate so that it is suspended out of the way of the work space.

7. Optionally, to check mechanical stop alignment, first attach the empty glass syringes to the mixer inlets. Note: Volumetric flowrates are varied using syringes of different barrel diameters, since the syringes are depressed simultaneously at the same linear velocity. The initial and final vertical heights must be the same for all syringes and can be adjusted using set screws tapped into the plunger shaft ${ }^{22}$. The mechanical stops ensure that excessive damage to the glass syringes does not occur.

1. Optionally, lower the mobile plate so that is comes to rest on the mechanical stops. Ensure that these are aligned so that the plate also comes to rest immediately before contacting the empty syringes (as seen in Figure 2).

2. Optionally, loosen the mechanical stops and reposition, if needed. Remove the glass syringes and reset the mobile plate out of the way.

Note: For operation with plastic syringes, the mechanical stops are not required. 
8. Place the open quench bath below the outlet tubing to collect the effluent.

9. Draw Solution A into a $1 \mathrm{~mL}$ gas-tight syringe using a blunt tip needle. Remove all air bubbles and dispose of the needle. Prime the solution to the end of the syringe luer fitting. Repeat this process for Solutions B and C.

10. Draw Solution $\mathrm{D}$ into a $2.5 \mathrm{~mL}$ gas-tight syringe using a blunt tip needle. Remove all air bubbles and dispose of the needle. Prime the solution to the end of the syringe luer fitting.

Note: These volumes have been selected so that the initial syringe plunger heights are the same. If volumes are changed, they still must meet this height requirement.

11. Assemble the four syringes onto the mixer in a clockwise fashion in alphabetical order. See Figure 1B for final appearance and syringe orientation schematic.

Note: Check that no syringe height is significantly different from the others and troubleshoot as needed.

3. Perform mixer operation and cleaning.

1. Grip the bearing housing on both sides of the mobile plate. Do not place fingers on the bottom face of the housing because this is a pinch hazard against the mechanical stops. Slowly lower the mobile plate so that it is resting evenly but barely touching the syringes.

2. Steadily and smoothly depress the plate, aiming to complete the operation in about $0.5-1 \mathrm{~s}$ for these stream volumes ${ }^{22}$.

1. Remove and cap the quench bath tube which now contains the NP dispersion.

3. Take the mixer with the syringes still attached and hold over a waste container. Remove the syringes, allowing the hold-up volume to drain into the container. Hold the mixer assembly upside and disassemble the mixer using the spanner wrench.

4. Using a spray bottle, rinse the outlet tubing with several milliliters of solvent (e.g., acetone) and dry with air or nitrogen.

5. Rinse the mixing geometry with a good solvent (e.g., deionized water or DMSO) and then rinse with acetone using several milliliters from a spray bottle. Dry with an air or nitrogen stream.

6. Rinse the O-ring in a stream of deionized water and blot dry.

7. Rinse the top disk thoroughly with several milliliters of acetone using a solvent bottle until visually clean. Dry with an air or nitrogen stream both the surface and the syringe fittings.

8. Rinse each syringe with several milliliters of a good solvent (e.g., deionized water or acetone) from a solvent bottle. Apply a final rinse of several milliliters of acetone and air dry before next use.

4. Perform post-processing and analysis.

1. Add $50 \mu \mathrm{L}$ of the calcium chloride dihydrate crosslinker solution dropwise while stirring at about $75 \%$ maximum speed.

2. Add $50 \mu \mathrm{L}$ of the ammonia solution dropwise while stirring at $75 \%$ maximum speed. Age for at least $30 \mathrm{~min}$.

3. Characterize the NP size as described in steps 2.3.1 and 2.3.2.

4. Process the NP dispersion to produce either microparticles or coated NPs as described in the literature $19,20,21$.

\section{Modifications for Formulation Scale-up}

1. Prepare the solvent and antisolvent solutions as described in steps 1,2 , or 3 at the desired composition and at sufficient volume for the required formulation size.

2. Optionally, if needed, clean and sterilize the mixer in place using a suitable protocol prior to NP formation.

Note: Sequential rinses of CIP 100, water (to neutral pH), CIP 200, water (to neutral pH), and a suitable solvent have been employed in the past. Additionally, sterile filters can be attached to the inlets of the mixer in instances where final particle size precludes sterilization by filtration.

3. Load the solutions into gas-tight syringes of suitable volume and attach polytetrafluoroethylene (PTFE) tubing with a luer adapter fitted on the end. Manually prime the solutions to the end of the tubing.

1. Load the syringes into a syringe pump and attach the syringes to the mixer inlets on either the CIJ or the MIVM, as required. Note: Alternatively, flow controllers can be used at lab or pilot scale to provide larger volume capabilities than a syringe pump.

Successful operation requires steady flow and sufficient pressure drop, which means that pressurized vessels with flow metering on the outlet are the most appropriate selection for large scale production.

4. Place a collection vessel containing a quench bath of sufficient volume, if required, beneath the outlet tubing.

5. Set the volumetric flow rates to match those achieved manually (e.g., about 30-60 $\mathrm{mL} / \mathrm{min}$ per stream).

Note: If using the CIJ, the pump flow rates must be identical. If using the MIVM, different inlets can have different flow rates.

6. Simultaneously begin the pumps. Collect about $5-10 \mathrm{~mL}$ of effluent as waste in a small vial (this is a "start-up volume") and then begin collecting in the quench bath.

7. Characterize and process as described in the corresponding formulation section above. 


\section{Representative Results}

Screening of NP formulations with FNP is rapid and requires small quantities of material (on the order of 1-10 mg). The FNP protocol to encapsulate hydrophobic compounds such as vitamin E (step 1) results in a stable, clear or lightly opalescent NP dispersion. Dynamic light scattering (DLS) provides a robust means to characterize the particle size. As shown in Figure 3, the process produces NPs with a low polydispersity in a reproducible fashion. The typical polydispersity index (PDI) is less than 0.20 , indicating a relatively monodisperse population. The PDI is obtained from the autocorrelation function and is often implemented into instrument software. It is a ratio of the second to the first moment, where values of 0.1 are generally obtained for monodisperse particles ${ }^{26}$. For the four vitamin E/PS- $b$-PEG formulation replicates reported, the value was $0.12 \pm 0.02$ and the average diameter was $107 \pm 7 \mathrm{~nm}$. A typical "misfire" due to either uneven depression of the syringes or slower depression speed is also reported in Figure 3. The polydispersity was unaffected, but the size was slightly larger (135 nm). Including this sample, the new metrics for particle size are $113 \pm 14 \mathrm{~nm}$. A misfire results in periods of time where the chamber contains only a single stream type. It is important that the entire stream experiences the same process history and relative volumes of the organic and aqueous streams within the mixer. Without a stabilizer, an opaque solution with visible aggregates is produced. The DLS autocorrelation function for this sample is non-monotonic and does not decay smoothly, as seen in the Figure 3 inset.

Particle size control by FNP is demonstrated in Figure 4, where varying the relative amounts of core material - poly(styrene) $1.8 \mathrm{k}$ in this case - and PS- $b$-PEG stabilizer resulted in particles sizes that ranged from 49-152 $\mathrm{nm}$. These particle sizes were generated with THF streams containing a total mass concentration of core and stabilizer of $20 \mathrm{mg} / \mathrm{mL}$, where $25 \%, 50 \%$, or $75 \%$ of the mass was the poly(styrene) core material. The polydispersity of the nanoparticles was always less than 0.15 . Extensive discussion of parameter effects on particle size produced by FNP may be found in the literature ${ }^{10}$. The loading can be tuned by holding the solvent volume constant and varying the relative volumes of the core and stabilizer stock solutions. Similarly, the total mass concentration can be varied by preparing stock solutions at values other than 10 $\mathrm{mg} / \mathrm{mL}$. Under certain conditions, it is possible to observe an empty micelle population by DLS ${ }^{27}$. This does not have any detrimental effect other than broadening the measured particle size distribution. When the sizes are similar, this may manifest as a single broad peak rather than two separate peaks.

The same CIJ mixer can also be used to encapsulate hydrophilic compounds by iFNP, as exemplified in step 2 of the Protocol. The particles produced in the reported formulation are around $65 \mathrm{~nm}$ with a low polydispersity of 0.08 . The size distribution can be seen in Figure $\mathbf{5 A}$ (dashed lines). The effect of crosslinking the PAA carboxylic acid residues on particle stability is demonstrated by DLS analysis in a strong solvent such as DMSO, as shown in Figure 5B. The autocorrelation function for well-crosslinked particles should start near a value of 1 and drop off sharply to 0 at a characteristic time that is related to the particle size (solid line). Particles that swell extensively or dissolve are not crosslinked and show minimal autocorrelation signal (dotted line). For iFNP, failed trials manifest in similar ways as described for FNP above. Visible aggregates may be seen or poor DLS autocorrelation function shape may be observed. The MIVM can be used for FNP or iFNP when more than two inlet streams are required due to system constraints such as solubility or chemical incompatibility. A small-scale version of the MIVM (the $\mu$ MIVM) with its mixer stand is shown in Figure 2. As with the CIJ, this mixer can be used to encapsulate hydrophobic or hydrophilic compounds ${ }^{22}$. In step 3 , a protocol for the encapsulation of a hydrophilic protein, OVA, by iFNP was described. The particle size distribution is shown in Figure 5A (solid line). The size is around $125 \mathrm{~nm}$ with a PDI of 0.16 . A general protocol for syringe pump operation at larger scales is provided in step 4. 
(A)

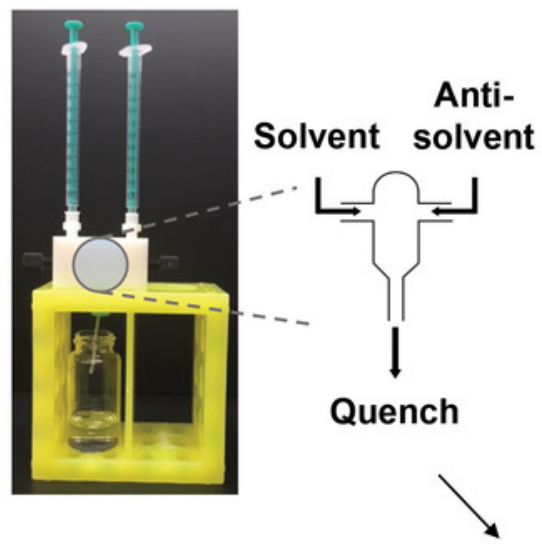

(B)

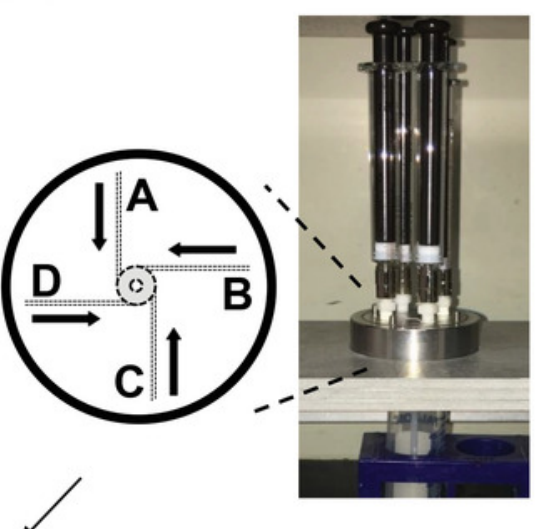

(C)

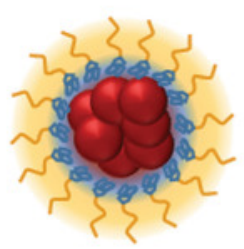

Figure 1: Mixer assembly and internal flow pattern schematics. (A) The confined impinging jets $(\mathrm{ClJ})$ mixer with attached syringes is positioned above the quench bath. Not pictured are a stir bar in the quench bath vial and a stir plate. The mixing geometry is depicted in the expanded view showing the two stream inlets that impinge in the center of the chamber. (B) A multi-inlet vortex mixer (the $\mu M I V M$ ) is shown with glass syringes and positioned in the stand above a quench bath. The mobile plate and the mechanical stops have been cropped from the picture. The expanded view shows the vortex chamber and the inlet channels schematically. (C) A schematic representation of core-shell NPs produced by FNP. Red spheres represent the therapeutic which, combined with the blue collapsed polymer block, comprise the NP core. The yellow polymer block forms the brush layer imparting steric stabilization to the NPs. Please click here to view a larger version of this figure.

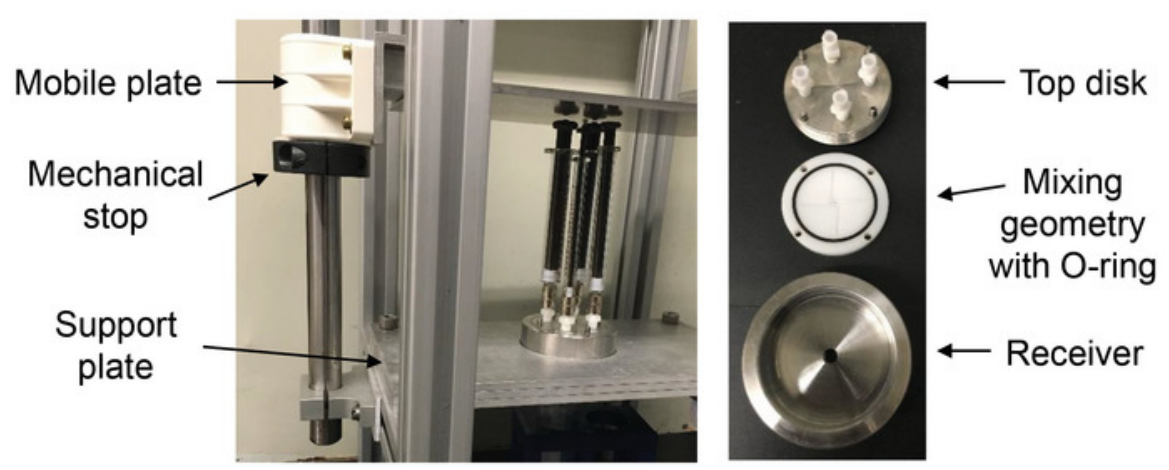

Figure 2: $\mu$ MIVM terminology and components for assembly. The $\mu$ MIVM requires a mixer stand to enable uniform depression of the four syringes. In this case, the syringe plunger heights must all be uniform to ensure consistent mixing. It can alternatively be operated using syringe pumps. The mixer stand with labeled components is shown at left of the figure. On the right is the disassembled mixer with the O-ring in place on the mixing geometry disk. Please click here to view a larger version of this figure. 




Figure 3: Particle size distribution of polymeric nanoparticles containing a core of vitamin E and stabilized by PS-b-PEG. Dynamic light scattering (DLS) provides intensity-weighted size distributions that indicate the NP diameter distribution. Curves are the average of triplicate analyses for each trial and have been rescaled to produce identical maximum peak heights. The four replicates (solid lines) indicate the high reproducibility of the method (standard deviation $=7 \mathrm{~nm}$ ). Also included is a representative misfire (dashed line), such as slower syringe speed or uneven depression of the two syringes, which results in larger particle diameter. The standard deviation of the NP size including the misfire was $14 \mathrm{~nm}$. (Inset) Without the PS- $b$-PEG stabilizer, large micron-scale aggregates (or droplets, in the case of an oil like vitamin E) are formed. The DLS autocorrelation function of a run without the stabilizer (dotted line) is shown along with a representative autocorrelation from a nanoparticle replicate (solid line). The autocorrelation function shows a number of characteristic timescales for the control sample, indicating a polydisperse population. Please click here to view a larger version of this figure.

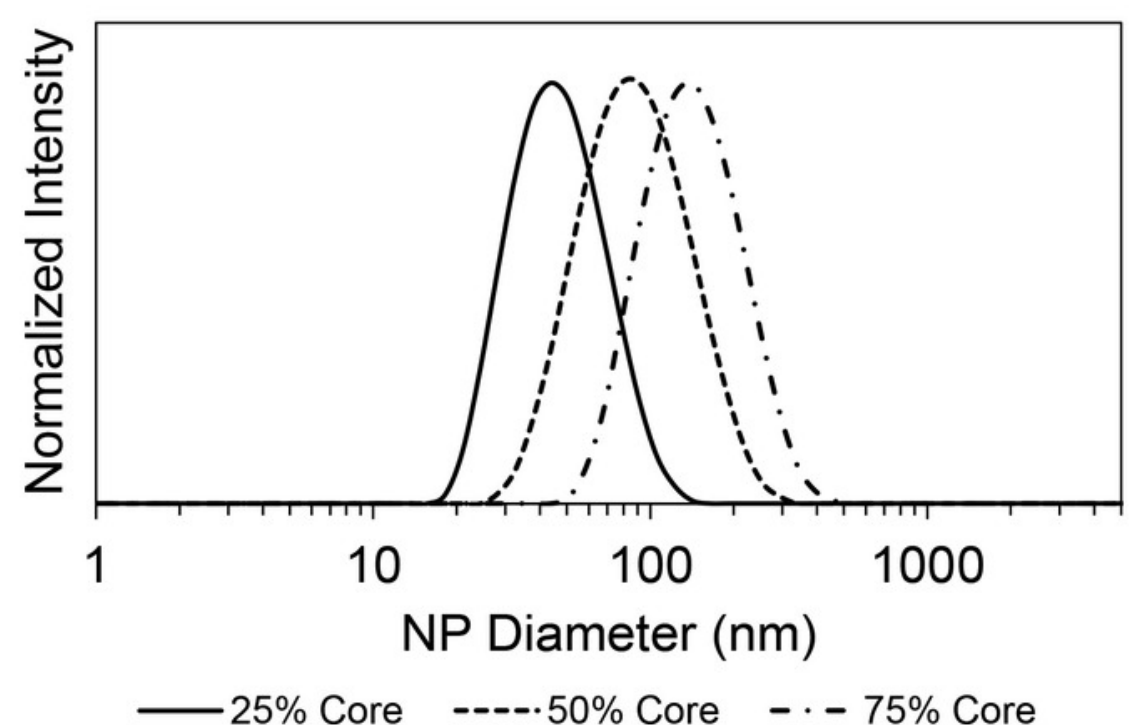

Figure 4: Particle size control by FNP through varying relative ratios of core material to stabilizer. The intensity-weighted size distributions of three formulations with a poly(styrene) core stabilized by PS- $b$-PEG are depicted. The total mass concentration in THF was $20 \mathrm{mg} / \mathrm{mL}$ and the antisolvent was water. The formulations were prepared in a ClJ mixer. The fraction of the mass composed of the core material is listed in the legend. For example, the $25 \%$ core sample contained $5 \mathrm{mg} / \mathrm{mL}$ poly(styrene) and $15 \mathrm{mg} / \mathrm{mL}$ PS- $b$-PEG. The average sizes for the $25 \%$ (solid line), $50 \%$ (dashed line), and $75 \%$ (mixed dash line) core loadings were $49 \mathrm{~nm}, 96 \mathrm{~nm}$, and $152 \mathrm{~nm}$, respectively. All PDI values were less than 0.15 . Please click here to view a larger version of this figure. 
(A)

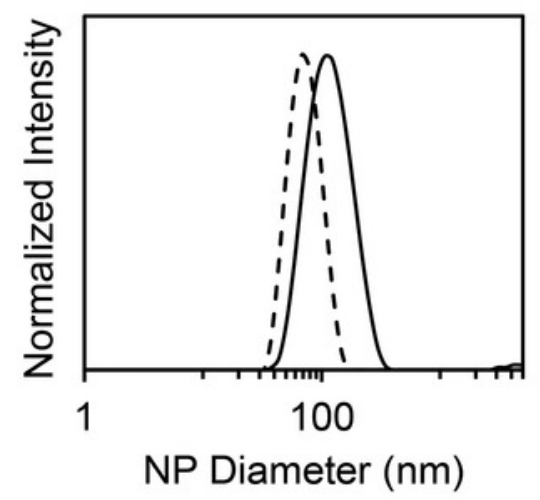

(B)

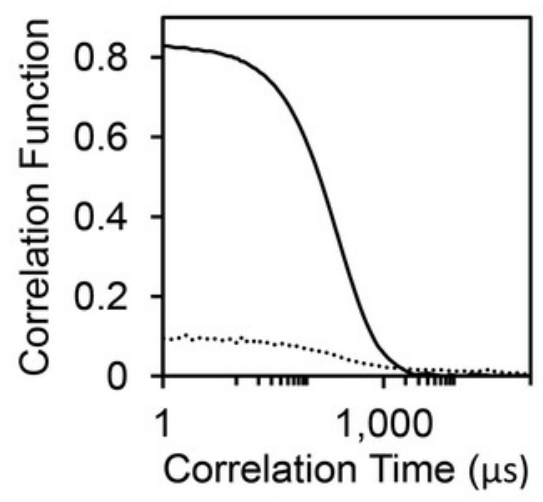

Figure 5: Characterization of inverted NPs made in a CIJ mixer or $\mu$ MIVM. (A) DLS curves are the average of triplicate analyses for each formulation. The dashed line indicates the size distribution of $3 \mathrm{k}$ MD particles made in the CIJ mixer while the solid line is the size distribution of OVA particles made in the $\mu$ MIVM. (B) The strength of crosslinking can be assessed by DLS using DMSO as the diluent. The DLS autocorrelation function indicates the strength of crosslinking through the initial autocorrelation value and the observation of a clean transition to a value of zero. The dashed line depicts the autocorrelation function for a particle with no crosslinker showing a weak initial signal and a broad decay time. The solid line depicts the autocorrelation after addition of a strong crosslinker (in this case, tetraethylenepentamine), which shows a strong initial signal and a defined decay timescale. Please click here to view a larger version of this figure. 

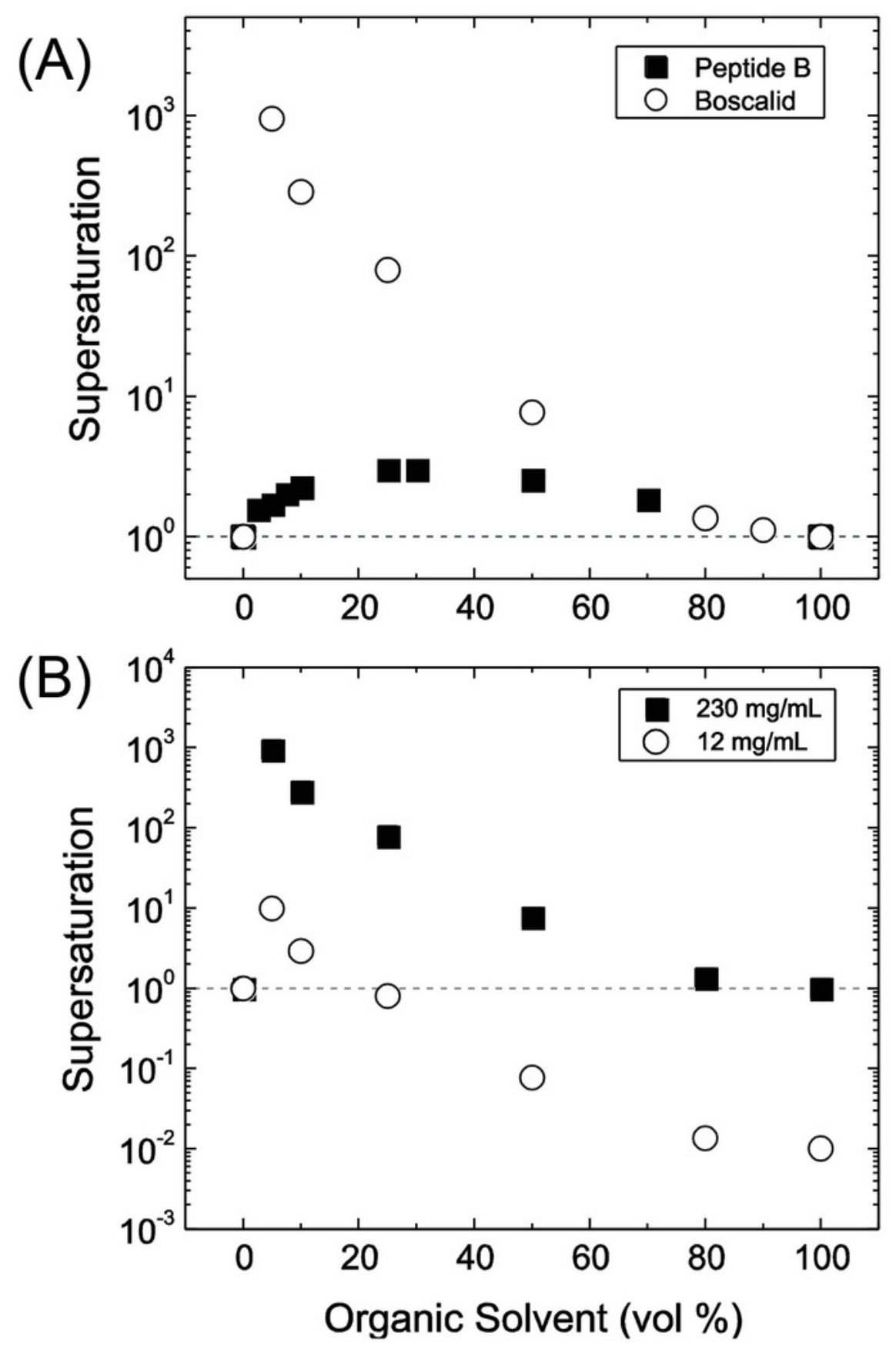

Figure 6: Supersaturation, $\mathrm{S}$, as a function of the relative mixing ratios of organic solvent to water. (A) Comparison of highest attainable supersaturation for $(\circ)$ boscalid, a pesticide, and $(\boldsymbol{m})$ peptide $B$, a seven-residue model peptide. The organic stream contains boscalid at a concentration of $230 \mathrm{mg} / \mathrm{mL}$ and peptide $B$ at $200 \mathrm{mg} / \mathrm{mL}$, their saturation concentrations. There is a maximum supersaturation that depends on each active pharmaceutical ingredient (API)/solvent system. (B) When the concentration of boscalid in the organic stream is decreased 20 fold, the conditions at which supersaturation and nanoprecipitation are achieved become limited. This figure is reprinted with permission from Elsevier ${ }^{9}$. Please click here to view a larger version of this figure.

Supplemental Files. Please click here to download the files.

\section{Discussion}

The encapsulation of hydrophobic compounds such as vitamin E, as in step 1 of the Protocol, has been extensively described ${ }^{9,14,28}$. Relatively monodisperse particles are produced because the time scale for mixing is shorter than the time scale for the aggregation and growth of the particles. Specifically, the mixed solvent/antisolvent solution rapidly becomes homogeneous, which enables nucleation to occur uniformly. Assembly of the block copolymer to the particle surface then provides steric stabilization that halts particle growth ${ }^{5}$. Since mixing time in the 
chamber (turbulence) is a function of the inlet flow rates to the CIJ or the MIVM, there is an inlet rate, which occurs after the transition to turbulent mixing, where the particle size is essentially constant ${ }^{13}$. This provides additional robustness to the process as some batch-to-batch variation in inlet flowrate (i.e., syringe depression speed) can be tolerated without significant impact to the final NP size as seen from Figure 3. Slower or uneven inlet speeds can result in larger particles or more polydisperse distributions, as seen for the misfire example. FNP has also been extended to encapsulate hydrophilic compounds in nanoparticles by inverse Flash NanoPrecipitation. These inverted nanoparticles can then be used to create microparticles or be coated with PEG to create water-dispersible nanoparticles ${ }^{25}$. The underlying assembly principles remain the same, though there is the added complexity of crosslinking the particle core. This is necessary for stabilization of the particle in an aqueous environment. In general, a 1:1 charge ratio compared to the polyacid block is sufficient, though the ionic interactions can be promoted by $\mathrm{pH}$ adjustment through the addition of a base ${ }^{19}$. In this protocol, only the first process step to form inverted NPs has been described.

In addition to fast mixing, successful formulation by FNP or iFNP is limited to instances where several conditions can be met ${ }^{9,14}$. First, all stream inputs must be miscible. While emulsions have been used to produce NPs, FNP requires a uniform solution phase in the mixer. Second, the core component must be nearly insoluble at the solvent conditions in the mixer (for the $\mathrm{ClJ}$, a 50/50 mixture by volume) to drive rapid nucleation. Otherwise, a significant portion will remain unencapsulated or will precipitate after further dilution with antisolvent. The MIVM can enable higher antisolvent content in the mixing chamber to address core material solubility limitations. It is often useful to generate supersaturation curves from solubility data as a function of solvent composition to guide process design ${ }^{9}$. Figure 6 shows representative curves for two compounds. Low supersaturation at the mixing chamber conditions merits operating at different compositions, typically using the MIVM. Higher supersaturation favors the nucleation of the core component over particle growth but a mismatch in assembly time of the core material and the stabilizer can result in large aggregates of the therapeutic. D'Addio and Prud'homme have reviewed the application of such supersaturation curves in detail ${ }^{9}$. Finally, the BCP must be molecularly dissolved in the solvent stream and the antisolvent stream must be selective for one block. The BCP must be sufficiently amphiphilic to provide both a solvophobic driving force from the collapsed block to anchor the stabilizer on the particle surface and for the solvated block to impart steric stability to the particle. Solvents other than those described in the protocol may be used as long as they meet these constraints.

Practice with manual syringe operation can improve the success rate during screening. As noted above, operation above the transition to homogeneous, turbulent mixing conditions means that small variations in flow rate are tolerated in the process ${ }^{28}$. Scale-up to pump-driven, computer-controlled flows results in even greater gains in consistency due to the reproducible inlet flow rates. At any point during postprocessing of the particles, visual inspection or DLS analysis may indicate the presence of large aggregates which can be due to incidental dust or particle instability. When necessary, the stream can be filtered with an appropriate filter pore size. In the absence of aggregates, we have found that less than $5 \%$ mass is typically lost when filtering PEG-coated nanoparticles if the nominal filter size is larger than the particle size distribution. When filtering aggregates, experimental determination of mass lost during the process is necessary. Quantification of the mass loss can be carried out in one of two ways. The total solids mass in a given volume can be determined by thermogravimetric analysis before and after filtration to identify the extent of change (see Supplemental Information Section 2). Alternatively, the particles can be recovered (e.g., by lyophilization) and dissolved in a good solvent. The concentration of the core material can then directly be measured by an appropriate technique such as ultraviolet-visible spectrophotometry or chromatography.

For FNP, the residual 10 vol\% organic solvent (e.g., THF) must be removed from the aqueous dispersion. This can be done by evaporative distillation ${ }^{14,29}$, dialysis ${ }^{30}$, or tangential flow filtration ${ }^{31,32}$. Practical considerations for each processing step are described in the citations provided. For dialysis, typical membranes are $3.5 \mathrm{kDa}$ or 6-8 kDa cutoffs, though larger options are available. This molecular weight cutoff is sufficient for solvent removal when dialyzed for $24 \mathrm{~h}$ using several bath changes. The use of tangential flow filtration entails some process development as care must be taken to avoid inducing aggregation due to concentration polarization at the membrane surface. We have found that reducing the organic solvent composition below a system-dependent value, usually $2-10$ vol\%, eliminates aggregation at the membrane surface. After processing, the concentration of nanoparticles is readily determined by thermogravimetric analysis (see Supplemental Information Section 2). It is often desirable to transport or store particles in a highly stable form. Aqueous dispersions can simply be frozen rapidly using a dry ice/ acetone mixture and then stored at $-80^{\circ} \mathrm{C}$. Alternatively, dry powders can be obtained by lyophilization ${ }^{33,34}$ or spray drying $^{24}$. Frequently, a cryoprotectant must be added to reduce nanoparticle aggregation during freezing or drying. Sugars (sucrose, trehalose, etc.), poly(ethylene glycol), or cyclodextrins can be screened for effectiveness over a range of concentrations by monitoring size by DLS ${ }^{35,36,37,38}$. Common NP stability problems during processing are often related to solubility or phase separation in the core resulting in rearrangement towards a lower energy state under conditions where mobility is increased. Use of co-core materials, alternative stabilizers, or modified external solution composition can help improve stability ${ }^{14,16,17,39,40,41}$.

As noted above, the MIVM enables higher antisolvent content in the mixing chamber when required to achieved high supersaturation. It can also allow for the physical segregation of species into more than two streams when reactivity or solubility constraints demand it. An example is the formation of zein protein-stabilized nanoparticles of the antibiotic clofazimine ${ }^{24}$. The hydrophobic clofazimine is introduced in an acetone stream; zein is introduced in a $60 \%$ ethanolic aqueous stream; casein, which complexes with zein, is brought in with an aqueous buffer stream, and the fourth stream is additional buffer to increase the ratio of water to acetone and ethanol. Two solvent streams are required since clofazimine and zein are not soluble in a common solvent. This process could not be accomplished in a two-jet CIJ mixer. This protein-stabilized formulation also demonstrates that FNP is not limited to BCP stabilizers. Janus particles have been produced without stabilizer ${ }^{42}$ and a range of low-cost stabilizers have been demonstrated for oral applications ${ }^{24}$. Notably, copolymers such as hydroxypropyl methylcellulose can be used in lieu of block copolymers ${ }^{24}$. Core materials can be made more hydrophobic by a number of techniques. Hydrophobic ion pairing has been applied to encapsulate a wide range of compounds that have intermediate solubility ${ }^{43,44,45}$. Extremely hydrophobic prodrugs have been generated and then encapsulated $^{46}$. Nucleic acids have been encapsulated through complexation with cationic lipids ${ }^{47}$. Importantly, these studies have shown that FNP can produce a range of particle surface chemistries. Further, mixed stabilizers containing a fraction of BCP that has been modified with a targeting ligand on the chain end have been used. This enables precise control over ligand content on the surface since particle composition reflects the input stream composition ${ }^{23,48}$. Similarly, it is possible to incorporate multiple core components as well, including dyes and inorganic nanoparticles ${ }^{3,8}$.

Flash NanoPrecipitation is a scalable approach to polymeric nanoparticles comprised of either a hydrophobic or a hydrophilic core. If the criteria enumerated above are met, generally over $95 \%$ of the core material is encapsulated at high mass fraction in the particle. The three examples presented here were carried out at bench scale, requiring a few milligrams of material and about $0.5 \mathrm{~mL}$ in each inlet stream. This allows for 
rapid screening of particle conditions for formulation optimization. Scale-up of lead formulations to larger batch sizes is a matter of running the process for longer, which can readily be accomplished through the use of syringe pumps or flow controllers. By contrast, the scale-up of bulk addition nanoprecipitation faces well-documented challenges in maintaining sufficient micromixing at the point of addition and accounting for the effect of changing vessel geometry ${ }^{49}$. This is a major barrier, since it is crucial to manufacture particles in a consistent manner to meet FDA requirements ${ }^{50}$. Microfluidics techniques can also produce uniform, reproducible nanoparticles, but only enable production in the milligram range. For example, Karnik et al. reported production rates of $0.25 \mathrm{mg} / \mathrm{min}$ for a drug release study ${ }^{51}$. Further scale-up typically entails parallelization at high capital cost ${ }^{12}$. With FNP, it is straightforward to produce $1 \mathrm{gram}$ of nanoparticles at $600 \mathrm{mg} / \mathrm{min}$ with a syringe pump and a few fittings to connect to the mixer inlets. Consequently, FNP represents both an accessible lab-scale screening tool as well as a scalable approach to NP production for translational work.

\section{Disclosures}

The authors have nothing to disclose.

\section{Acknowledgements}

This work was supported by funding from Optimeos Life Sciences, the National Science Foundation (CBET 1605816), the Bill and Melinda Gates Foundation (BMGF, OPP1150755), and the National Science Foundation Graduate Research Fellowship (DGE-1656466) awarded to K.D.R.

\section{References}

1. Bobo, D., Robinson, K. J., Islam, J., Thurecht, K. J., Corrie, S. R. Nanoparticle-Based Medicines: A Review of FDA-Approved Materials and Clinical Trials to Date. Pharmaceutical Research. 33 (10), 2373-2387, (2016).

2. D'Mello, S. R. et al. The evolving landscape of drug products containing nanomaterials in the United States. Nature Nanotechnology. 12 (6), 523-529, (2017).

3. Gindy, M. E., Prud'homme, R. K. Multifunctional nanoparticles for imaging, delivery and targeting in cancer therapy. Expert Opinion on Drug Delivery. 6 (8), 865-878, (2009).

4. Chen, G., Roy, I., Yang, C., Prasad, P. N. Nanochemistry and Nanomedicine for Nanoparticle-based Diagnostics and Therapy. Chemical Reviews. 116 (5), 2826-2885, (2016).

5. Johnson, B. K., Prud'homme, R. K. Mechanism for Rapid Self-Assembly of Block Copolymer Nanoparticles. Physical Review Letters. 91 (11), 118302-118302, (2003)

6. Schubert, S., Delaney, J. J. T., Schubert, U. S. Nanoprecipitation and nanoformulation of polymers: from history to powerful possibilities beyond poly(lactic acid). Soft Matter. 7 (5), 1581-1588, (2011).

7. Lebouille, J. G. J. L., Stepanyan, R., Slot, J. J. M., Cohen Stuart, M. A., Tuinier, R. Nanoprecipitation of polymers in a bad solvent. Colloids and Surfaces A: Physicochemical and Engineering Aspects. 460, 225-235, (2013).

8. Akbulut, M. et al. Generic method of preparing multifunctional fluorescent nanoparticles using flash nanoPrecipitation. Advanced Functional Materials. 19 (5), 718-725, (2009).

9. D'Addio, S. M., Prud'homme, R. K. Controlling drug nanoparticle formation by rapid precipitation. Advanced Drug Delivery Reviews. 63 (6), 417-426, (2011).

10. Pagels, R. F., Edelstein, J., Tang, C., Prud'homme, R. K. Controlling and Predicting Nanoparticle Formation by Block Copolymer Directed Rapid Precipitations. Nano Letters. 18 (2), 1139-1144, (2018).

11. Ding, S., Anton, N., Vandamme, T. F., Serra, C. A. Microfluidic nanoprecipitation systems for preparing pure drug or polymeric drug loaded nanoparticles: an overview. Expert Opinion on Drug Delivery. 13 (10), 1447-1460, (2016).

12. Valencia, P. M., Farokhzad, O. C., Karnik, R., Langer, R. Microfluidic technologies for accelerating the clinical translation of nanoparticles. Nature Nanotechnology. 7 (10), 623-629, (2012).

13. Johnson, B. K., Prud'homme, R. K. Chemical processing and micromixing in confined impinging jets. AlChE Journal. 49 (9), $2264-2282$, (2003).

14. Saad, W. S., Prud'homme, R. K. Principles of nanoparticle formation by flash nanoprecipitation. Nano Today. 11 (2), 212-227, (2016).

15. Han, J. et al. A simple confined impingement jets mixer for flash nanoprecipitation. Journal of Pharmaceutical Sciences. 101 (10), 4018-4023, (2012).

16. Kumar, V., Wang, L., Riebe, M., Tung, H. H., Prud'homme, R. K. Formulation and stability of itraconazole and odanacatib nanoparticles: Governing physical parameters. Molecular Pharmaceutics. 6 (4), 1118-1124, (2009).

17. Liu, Y., Kathan, K., Saad, W., Prud'homme, R. K. Ostwald Ripening of $\beta$-Carotene Nanoparticles. Physical Review Letters. 98 (3), 036102-036102, (2007).

18. Liu, Y., Cheng, C., Liu, Y., Prud'homme, R. K., Fox, R. O. Mixing in a multi-inlet vortex mixer (MIVM) for flash nano-precipitation. Chemical Engineering Science. 63, 2829-2842, (2008).

19. Pagels, R. F., Prud'homme, R. K. Polymeric nanoparticles and microparticles for the delivery of peptides, biologics, and soluble therapeutics. Journal of Controlled Release. 219, 519-535, (2015).

20. Pagels, R. F., Prud'homme, R. K. in Control of Amphiphile Self-Assembling at the Molecular Level: Supra-Molecular Assemblies with Tuned Physicochemical Properties for Delivery Applications Vol. 1271 ACS Symposium Series. Ch. 11, 249-274 American Chemical Society (2017).

21. Markwalter, C. E., Prud'homme, R. K. in Control of Amphiphile Self-Assembling at the Molecular Level: Supra-Molecular Assemblies with Tuned Physicochemical Properties for Delivery Applications Vol. 1271 ACS Symposium Series. Ch. 12, 275-296 American Chemical Society (2017).

22. Markwalter, C. E., Prud'homme, R. K. Design of a Small-Scale Multi-Inlet Vortex Mixer for Scalable Nanoparticle Production and Application to the Encapsulation of Biologics by Inverse Flash NanoPrecipitation. Journal of Pharmaceutical Sciences. 107 (9), 2465-2471, (2018).

23. Gindy, M. E., Ji, S., Hoye, T. R., Panagiotopoulos, A. Z., Prud'Homme, R. K. Preparation of poly(ethylene glycol) protected nanoparticles with variable bioconjugate ligand density. Biomacromolecules. 9 (10), 2705-2711, (2008). 
24. Zhang, Y. et al. Design and Solidification of Fast-Releasing Clofazimine Nanoparticles for Treatment of Cryptosporidiosis. Molecular Pharmaceutics. 14 (10), 3480-3488, (2017).

25. Pagels, R. F. Polymeric Nanoparticles and Microparticles for the Delivery of Hydrophobic and Hydrophilic Therapeutics. Doctor of Philosophy thesis, Princeton University, (2018).

26. Frisken, B. J. Revisiting the method of cumulants for the analysis of dynamic light-scattering data. Applied Optics. 40 (24), 4087-4091, (2001).

27. Budijono, S. J., Russ, B., Saad, W., Adamson, D. H., Prud'homme, R. K. Block copolymer surface coverage on nanoparticles. Colloids and Surfaces A: Physicochemical and Engineering Aspects. 360 (1-3), 105-110, (2010).

28. Johnson, B. K., Prud'homme, R. K. Flash NanoPrecipitation of Organic Actives and Block Copolymers using a Confined Impinging Jets Mixer. Australia Journal of Chemistry. 56 1021-1024, (2003).

29. Kumar, V., Prud'homme, R. K. Nanoparticle stability: Processing pathways for solvent removal. Chemical Engineering Science. 64 (6), 1358-1361, (2009).

30. Shi, L., Shan, J., Ju, Y., Aikens, P., Prud'homme, R. K. Nanoparticles as delivery vehicles for sunscreen agents. Colloids and Surfaces A: Physicochemical and Engineering Aspects. 396, 122-129, (2012).

31. Dalwadi, G., Benson, H. A. E., Chen, Y. Comparison of Diafiltration and Tangential Flow Filtration for Purification of Nanoparticle Suspensions. Pharmaceutical Research. 22 (12), 2152-2162, (2005).

32. Pansare, V. J., Tien, D., Thoniyot, P., Prud'homme, R. K. Ultrafiltration of nanoparticle colloids. Journal of Membrane Science. 538, 41-49, (2017).

33. D'Addio, S. M. et al. Novel Method for Concentrating and Drying Polymeric Nanoparticles: Hydrogen Bonding Coacervate Precipitation Molecular Pharmaceutics. 7 (2), 557-564, (2010).

34. Abdelwahed, W., Degobert, G., Stainmesse, S., Fessi, H. Freeze-drying of nanoparticles: Formulation, process and storage considerations. Advanced Drug Delivery Reviews. 58 (15), 1688-1713, (2006).

35. Correa, S. et al. Highly Scalable, Closed-Loop Synthesis of Drug-Loaded, Layer-by-Layer Nanoparticles. Advanced Functional Materials. 26 (7), 991-1003, (2016)

36. Figueroa, C. Engineering Nanoparticles for Pharmaceutical Applications: Formulation and Freeze-drying Techniques. Doctor of Philosophy thesis, Princeton University, (2014).

37. Harada, A., Li, J., Kamachi, M. Preparation and properties of inclusion complexes of polyethylene glycol with alpha-cyclodextrin. Macromolecules. 26 (21), 5698-5703, (1993).

38. Troiano, G., Song, Y.-H., Zale, S, Wright, J., Van Geen Hoven, C. Stable Formulations for Lyophilizing Therapeutic Particles. United States patent (2013)

39. Kumar, V., Adamson, D. H., Prud'homme, R. K. Fluorescent polymeric nanoparticles: Aggregation and phase behavior of pyrene and amphotericin B molecules in nanoparticle cores. Small. 6 (24), 2907-2914, (2010).

40. Budijono, S. J. et al. Synthesis of stable block-copolymer-protected NaYF4:Yb3+, Er3+up-converting phosphor nanoparticles. Chemistry of Materials. 22 (2), 311-318, (2010).

41. Chen, T. et al. Protected peptide nanoparticles: Experiments and brownian dynamics simulations of the energetics of assembly. Nano Letters. 9 (6), 2218-2222, (2009).

42. Sosa, C. et al. Soft Multifaced and Patchy Colloids by Constrained Volume Self-Assembly. Macromolecules. 49 (9), 3580-3585, (2016)

43. Pinkerton, N. M. et al. Formation of stable nanocarriers by in situ ion pairing during block-copolymer-directed rapid precipitation. Molecular Pharmaceutics. 10 (1), 319-328, (2013).

44. Lu, H. D., Rummaneethorn, P., Ristroph, K. D., Prud'homme, R. K. Hydrophobic lon Pairing of Peptide Antibiotics for Processing into Controlled Release Nanocarrier Formulations. Molecular Pharmaceutics. 15 (1), 216-225, (2018).

45. Lu, H. D. et al. Encapsulation of OZ439 into Nanoparticles for Supersaturated Drug Release in Oral Malaria Therapy. ACS Infectious Diseases. 4 (6), 970-979, (2018).

46. Ansell, S. M. et al. Modulating the Therapeutic Activity of Nanoparticle Delivered Paclitaxel by Manipulating the Hydrophobicity of Prodrug Conjugates. Journal of Medicinal Chemistry. 51 (11), 3288-3296, (2008).

47. Gindy, M. E. et al. Mechanism of macromolecular structure evolution in self-assembled lipid nanoparticles for siRNA delivery. Langmuir. 30 (16), 4613-4622, (2014).

48. D'Addio, S. M. et al. Optimization of cell receptor-specific targeting through multivalent surface decoration of polymeric nanocarriers. Journal of Controlled Release. 168 (1), 41-49, (2013).

49. Perry's Chemical Engineers' Handbook. 19-20 McGraw-Hill (2007).

50. Torrice, M. Does nanomedicine have a delivery problem? ACS Central Science. 2 (7), 434-437, (2016).

51. Karnik, R. et al. Microfluidic Platform for Controlled Synthesis of Polymeric Nanoparticles. Nano Letters. 8 (9), 2906-2912, (2008). 